\title{
MODULA - Uma abordagem estruturada para a resolução de problemas, aplicada ao ensino de Matemática na Engenharia
}

\author{
MODULA - A structured approach for problem solving applied to \\ Mathematics teaching in Engineering
}

Mateus Mosca Viana mosca@unifor.br UNIFOR

\section{Ana Teresa de Araújo Farias \\ anateresa@unifor.br UNIFOR}

Francisco Antonio de Araújo e Souza prof.faraujo@unifor.br UNIFOR

\begin{abstract}
Resumo
O método para a resolução de problemas proposto neste artigo se fundamenta no raciocínio dedutivo e é conhecido como "Desenvolvimento de cima para baixo", ou "Top Down Development", satisfatoriamente utilizado na Ciência da Computação. Especificamente, trata-se de uma adaptação dessa mesma concepção com o foco direcionado para o ensino de Matemática em cursos de Engenharia. Tal proposição foi motivada pela observação, ao longo da práxis docente dos autores e de pesquisas analisadas, sobre as dificuldades de estudantes na aprendizagem em Matemática. São descritos os procedimentos do método, bem como a modelagem matemática de um exemplo contextualizado, focando na realidade a ser modelada e adaptada ao estudo de situações matemáticas.

Palavras-chave: Matemática; Engenharia; Didática; Método de modelagem.

Abstract

The method for problem solving proposed in this article is based on deductive reasoning and is known as "Top Down Development" satisfactorily used in Computer Science. Specifically, it is an adaptation of this same conception with the focus directed to the teaching of Mathematics in Engineering courses. This proposition was motivated by the observation, throughout the teacher praxis of the authors and of analyzed research, on the difficulties of learning in Mathematics of students. The procedures of the method are described, as well as the mathematical modeling of a contextualized example, focusing on the reality to be modeled and adapted to the study of mathematical situations.
\end{abstract}

Keywords: Mathematics; Engineering; Didactics; Modeling method.

\section{Introdução}

Os diversos conhecimentos com os quais as pessoas têm contato, que fazem parte da sua vida cotidiana, são utilizados para a resolução dos mais variados problemas. Os exemplos são numerosos e tal é o caso da Engenharia, da Medicina, do Direito, por exemplo, apenas para citar três dos mais conhecidos.

Em qualquer desses conhecimentos apontados anteriormente, ou em qualquer outro que possa ser apresentado, devese ter em mente que sempre existe uma ciência básica para dar o suporte necessário ao seu funcionamento, e disso decorre a correta aplicação nas resoluções de problemas.

Com efeito, a Física é a ciência básica de onde a Engenharia retira os princípios utilizados para construir os seus artefatos. A Medicina, por sua vez, tem a sua prática assentada em conhecimentos da Biologia, assim como o exercício do Direito é fundamentado na Filosofia. Essas ciências básicas citadas apresentam focos específicos de aplicação. No entanto, existe outra ciência básica cuja atuação se manifesta de modo generalizado pelos diversos ramos do conhecimento, a saber: a Matemática.

A contribuição da Matemática à construção do conhecimento em todas as outras áreas aparece na forma do desenvolvimento de modelos, por meio dos quais é possível explicar o funcionamento dos mais variados fenômenos naturais. 
Conforme os paradigmas da Ciência moderna, que constituíram o Empirismo (Locke; Berkeley e Hume, 1975), "sabe-se que o Conhecimento Científico, em qualquer campo, considerando seus objetos de análise e processos naturais deve, de alguma forma, apoiar-se especialmente na experiência, na observação dos fatos e não somente na razão pura".

Desde aquele momento, uma questão ainda perdura: dado que qualquer experiência é necessariamente particular, ou seja, referente a objetos individuais, como pode essa experiência constituir base adequada para as leis científicas, que se referem ao universo inteiro de objetos? Ou o mesmo: como se pode passar de observações particulares para o caso geral? Essa questão epistemológica clássica é conhecida como "Questão da indução" (HUME, 1736).

Verifica-se, ao longo da História das Ciências e da Filosofia, que muito se tem refletido sobre a tarefa de resolver problemas, decorrendo daí variadas proposições de como deve ser o comportamento de quem procura por resolver problemas em qualquer campo de saber. Existe um trabalho clássico de George Polya, intitulado "How to Solve It", publicado originalmente em 1945, que tem atraído a atenção de gerações de estudiosos desse assunto.

Tomando por base Polya (1945) e outros autores citados, o objetivo deste artigo é apresentar recomendações metodológicas frente à necessidade de resolver um problema através de um modelo matemático. Não se pretende que tais recomendações se constituam em um método a ser seguido de modo intransigente. Ao contrário, elas permitirão que o interessado em resolver um problema de Matemática possa criar a sua própria estratégia de ação, qualquer que seja o cenário que se apresente. Essa linha de pensamento foi seguida na Ciência da Computação, como se pode ver em DROMEY (1987).

Considerando o título deste artigo, é fundamental diferenciar os conceitos "resolução" e "solução", pois enquanto uma é meio, a outra é fim. Assim "resolução" (como meio) é um procedimento para se chegar a uma demonstração racional, ou, melhor ainda, lógica, por deduções analisadas, de uma questão, problema ou decisão; enquanto que "solução" (como fim) é o encerramento ou resultado de proposições, suposições, ideias, ainda que não se tenha usado um raciocínio lógico para isso. A partir do exemplo apresentado será possível acompanhar o desenvolvimento do método proposto.

\section{A Problemática}

No contexto mundial da educação, muito se tem discutido sobre o desempenho, em todos os níveis, de estudantes de Matemática, especialmente após os ciclos avaliativos de larga escala, como o Programa Internacional de Avaliação de Estudantes (PISA) e, no Brasil, o Exame Nacional do Ensino Médio (ENEM) e o Exame Nacional de Desempenho de Estudantes (ENADE).

Após diversos ciclos avaliativos, com frequência, tem-se constatado o baixo desempenho dos estudantes em Matemática, mas, apenas isso. Raros são os desdobramentos dessas pesquisas de larga escala que efetivamente apresentem propostas para mitigar o baixo desempenho de estudantes, independente dos conhecimentos avaliados. Também raras são as pesquisas que buscam análises mais aprofundadas e localizadas, para além do mero diagnóstico, e que vêm dispensando qualquer prognóstico. Como exemplo dessas raridades (pelo menos no Brasil), destacam-se Oliveira (2016) e Almeida (2006).

Oliveira (2016, p. 475 e 476), articulando os processos de leitura, contagem e avaliação, afirma que a compreensão do desenvolvimento das habilidades relacionadas à leitura e contagem passa, necessariamente pela avaliação. Ainda conforme esse autor, por isso é fundamental saber que a avaliação possui três funções principais, que não se excluem mutuamente, conforme Hadji (1994, p. 34), certificar; regular e orientar, assim justificadas como:

A função de orientar corresponde às conclusões sobre o estágio em que se encontra a aquisição de conhecimentos para se aceder a outras aquisições. Também é vista como função preditiva e, por isso, teria como objetivo determinar, segundo Jorba e Sanmartí (2003, p. 27), "a situação de cada aluno antes de iniciar um determinado processo de ensinoaprendizagem, para poder adaptá-lo a suas necessidades". Para essa orientação, lança-se mão da modalidade diagnóstica da avaliação que pode ocorrer no início de cada situação de ensino-aprendizagem.

A função de regular está relacionada à orientação da ação educativa, quando se buscam as melhores estratégias para a facilitação a aprendizagem. Essa função, que corresponde à modalidade formativa da avaliação, equivale ao ajuste das ações no processo de ensinoaprendizagem, ficando mais evidenciada, por isso, durante o processo. Entretanto, há interfaces com as outras duas funções uma vez que ao se certificar e ao se orientar, procura-se 
MODULA - Uma abordagem estruturada para a resolução de problemas, aplicada ao ensino de Matemática na Engenharia

estabelecer um processo de ensino-aprendizagem que favoreça o aluno. Todas as informações e análises devem contribuir para isso.

A função de certificar, presente na modalidade somativa, considera a constatação sobre conhecimentos adquiridos, garantindo-se um título ou a passagem de um nível a outro (por exemplo, passagem do primeiro para o segundo ano após os exames finais e aferição das médias anuais). Tem-se em vista, ao focalizar-se essa função, observar se os alunos atingiram as exigências do sistema, mas, também, se alcançaram os pré-requisitos para aprendizagens posteriores.

Oliveira (2016, p. 475 e 476).

Observa-se, não apenas pelas críticas de Hadji, como também de diversos autores, como Demo, Luckesi, Hoffmann, Perrenoud, Saul, Bloom, Dias Sobrinho, entre outros, a inadequação dos termos "testar" e "medir", usados como sinônimo de avaliação, pois a expressão "medida", em educação, adquiriu uma conotação ampla e difusa. Não obstante esse viés, verifica-se que a avaliação educacional no Brasil e no mundo tem avançado significativamente em suas dimensões teóricas e metodológicas, mas, o que se pode dizer sobre a efetividade do processo de ensino e aprendizagem?

Neste estágio, os sistemas educacionais precisam estar em permanente análise e avaliação (metavaliação) e, para tanto, é fundamental buscar critérios para mensurar, com equidade, o desempenho de aprendizagem do estudante, o que depende de vários fatores, como se verifica a seguir.

Em pertinente pesquisa sobre o insucesso em Matemática na percepção de 52 professores de ensino fundamental e médio de escolas públicas e particulares do Distrito Federal, intitulada "Dificuldades de aprendizagem em Matemática e a percepção dos professores em relação a fatores associados ao insucesso nesta área" foi possível identificar que "As dificuldades de aprendizagem em Matemática podem ocorrer por diversos fatores, sejam eles afetivos, cognitivos ou mesmo físicos." (Almeida, 2006, p.12).

Considerando essa pesquisa e abstraindo os fatores relativos à conduta dos professores e à conduta dos estudantes, para concentrar-se tão somente nos fatores que levam ao insucesso em Matemática relativos aos métodos de ensino, verificou-se que:

Tabela 1 - O insucesso em Matemática e os métodos e técnicas de ensino (\%)

\begin{tabular}{|c|c|c|c|c|}
\hline $\mathrm{N}^{\mathrm{o}}$ & Proposição & 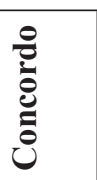 & 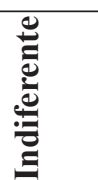 & 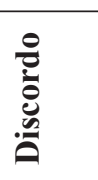 \\
\hline 2 & Os métodos de ensino são adequados à realidade do aluno. & 48,1 & 13,4 & 38,5 \\
\hline 7 & $\begin{array}{l}\text { Os programas curriculares são insuficientes para concretização no processo ensi- } \\
\text { no- aprendizagem }\end{array}$ & 64,7 & 13,8 & 21,5 \\
\hline 11 & A Matemática dada nas escolas não tem aplicação na vida real. & 38,4 & 3,9 & $\mathbf{5 7 , 7}$ \\
\hline 14 & Os programas de matemática estão em processo de transformação. & 48,0 & 36,6 & 15,4 \\
\hline 19 & $\begin{array}{l}\text { Os programas de Matemática estão fora da realidade dos alunos provocando } \\
\text { desinteresse. }\end{array}$ & $\mathbf{5 4 , 9}$ & 19,6 & 25,5 \\
\hline 20 & $\begin{array}{l}\text { A disciplina de matemática é vista como uma matéria que traz maus resultados } \\
\text { perante a sociedade. }\end{array}$ & 37,2 & 7,9 & 54,9 \\
\hline
\end{tabular}

Fonte: Almeida (2006, p. 6) com adaptações

Considerando o apurado por essa pesquisa, restam as seguintes questões: Se os métodos de ensino são adequados à realidade do aluno (Questão No. 2), como indica a maioria dos professores (48,1\%), e a Matemática dada nas escolas TEM 
aplicação na vida real (Questão No. 11), como indica a maioria dos professores (57,7\%), então o problema realmente está nos programas? (Questões 7, 14, 19). Não haveria um viés de corporativismo devido o método depender do professor, mas não o programa? Os estudantes confirmariam?

Se a disciplina de matemática NÃO é vista como uma matéria que traz maus resultados perante a sociedade, como indica a maioria dos professores $(54,9 \%)$, por que tem sido considerada uma disciplina "ofensiva", "obstáculo" e "coisa de gênio" no jargão popular e no senso comum?

Ainda segundo Almeida (2006, p. 2): “... as dificuldades de aprendizagem em Matemática (DAM), observa-se que existem muitas interrogações e, com frequência, não existe uma única causa que possa ser atribuída, mas sim várias delas conjuntamente. As causas das dificuldades podem ser buscadas no aluno ou em fatores externos, em particular no modo de ensinar a Matemática."

Assim, surge a seguinte questão norteadora: O que deve ser feito para organizar o trabalho pedagógico de forma a propiciar o desenvolvimento das competências e habilidades em Matemática?

\section{Referencial Teórico}

Os aspectos do método proposto têm origem em um contexto de práxis docente, no qual foram frequentemente observadas dificuldades no processo de ensino-aprendizagem de Matemática, abordado por Polya ainda nos anos 1940. Sendo o objeto central desse assunto ligado ao aprendizado de Matemática e suas dificuldades, encontra-se na área da Educação que, em última análise, pertence ao campo das Ciências Sociais Aplicadas. Desse modo, em qualquer proposição de novos métodos é necessário seguir o método científico, de modo a que seja possível validar as novas práticas reveladas.

O processo utilizado no método MODULA claramente se apresenta como uma aplicação da Teoria da Aprendizagem Significativa, de David Ausubel (1963), à proporção que permite ao aprendiz construir as suas estruturas mentais a partir de conhecimentos anteriores e é baseada nas seguintes dimensões da aprendizagem significativa:

Figura 1 - As dimensões da aprendizagem significativa

\begin{tabular}{|c|c|c|c|}
\hline \multirow{3}{*}{$\begin{array}{l}\text { - Aprendizagem } \\
\text { Significativa } \\
\text { Clarificação de relações } \\
\text { entre conceitos }\end{array}$} & $\begin{array}{l}\text { Instrução autotutorial bem } \\
\text { planejada }\end{array}$ & $\begin{array}{l}\text { Pesquisas científicas } \\
\text { (artes, ciências, etc) }\end{array}$ & \\
\hline & $\begin{array}{l}\text { Apresentações em livros } \\
\text { didáticos }\end{array}$ & Pesquisa escolar orientada & $\begin{array}{l}\text { Maior parte de "pesquisa } \\
\text { intelectual" }\end{array}$ \\
\hline & $\begin{array}{l}\text { Aulas teóricas de um modo } \\
\text { geral }\end{array}$ & $\begin{array}{l}\text { Trabalho escolar de } \\
\text { laboratório }\end{array}$ & $\begin{array}{l}\text { Produção intelectual de } \\
\text { rotina }\end{array}$ \\
\hline \multirow[t]{2}{*}{$\begin{array}{l}\text { Aprendizagem } \\
\text { Mecânica }\end{array}$} & $\begin{array}{l}\text { Tabelas (tabuadas) das } \\
\text { operações }\end{array}$ & $\begin{array}{l}\text { Aplicação de fórmulas ao } \\
\text { resolver problemas }\end{array}$ & $\begin{array}{l}\text { Solução de quebra- } \\
\text { cabeça por ensaio e erro }\end{array}$ \\
\hline & $\begin{array}{l}\text { Aprendizagem } \\
\text { Receptiva }\end{array}$ & & $\begin{array}{l}\text { Aprendizagem por } \\
\text { Descoberta }\end{array}$ \\
\hline
\end{tabular}

Fonte: Moreira, 1999.

Para Ausubel (1963, p. 58), “a aprendizagem significativa é o mecanismo humano, por excelência, para adquirir e armazenar a vasta quantidade de ideias e informações representadas em qualquer campo de conhecimento".

Também é possível considerar que processo utilizado no método MODULA para aprendizagem significativa tem origem vygotskyana, pois, para Vygotsky $(1987,1988)$, o desenvolvimento cognitivo não pode ser entendido sem referência ao contexto social, histórico e cultural em que ocorre. Para ele, os processos mentais superiores (pensamento, linguagem, comportamento voluntário) têm sua origem em processos sociais e o desenvolvimento cognitivo é a conversão de relações sociais em funções mentais. Nesse processo, toda relação/função aparece duas vezes, primeiro em nível social e, depois, 
MODULA - Uma abordagem estruturada para a resolução de problemas, aplicada ao ensino de Matemática na Engenharia

em nível individual. Primeiro entre pessoas (interpessoal, interpsicológica) e, após, no interior do sujeito (intrapessoal, intrapsicológica).

\section{Método}

O enunciado do problema proposto nesta seção teve origem em um caso real na construção civil. Apesar de ser uma situação simples e específica, servirá como exemplo para o entendimento do método proposto, pois permite a observação de aspectos importantes inerentes ao uso dele.

Assim, o estudo de caso apresentado neste artigo tem como pressupostos teóricos as concepções de YIN (2001), que enuncia:

“A utilização da teoria, ao realizar estudos de caso, não apenas representa uma ajuda imensa na definição do projeto de pesquisa e na coleta de dados adequados, como também se torna o veículo principal para a generalização dos resultados do estudo de caso". (p.54).

\subsection{O Problema}

Cenário

- Na obra de um edifício residencial com estrutura de concreto moldada no local e alvenaria de blocos de concreto, está sendo planejado um serviço de revestimento em uma parede. O mesmo será executado em pasta de gesso e feito com uma única camada. As dimensões da parede são $3 \mathrm{~m}$ de altura por $10 \mathrm{~m}$ de comprimento.

Dados técnicos

- Para a confecção da pasta, deverá ser utilizado o gesso em pó, com as seguintes características:

- Densidade aparente: $0,84 \mathrm{~kg} / \mathrm{dm}$

- Densidade absoluta: $2,7 \mathrm{~kg} / \mathrm{dm}$

- Água de amassamento: 0,45 (4501/m )

- Coeficiente de rendimento

$(\mathrm{CR}=($ densidade aparente/Densidade absoluta $)+$ Água de amassamento $)$

Sabe-se que

- Volume do pó = massa/(densidade aparente)

- Volume do aglomerante $=$ Volume da pasta/CR

Questão

- Qual deverá ser a espessura da camada de gesso sabendo que não se poderá usar mais do que cinquenta sacos de gesso, cada um com massa de $25 \mathrm{~kg}$ ?

\subsection{A aplicação do método proposto}

O fundamento do método proposto tem por base a formulação de três questões norteadoras, a saber:

\section{O que deve ser feito?}

Como deve ser feito?

De que maneira implementar?

O conjunto das respostas obtidas como resultado da formulação de cada pergunta se constituirá em um roteiro, destinado a desenvolver a resolução do problema proposto. A fim de facilitar o uso do método, é recomendável que tais respostas sejam representadas de acordo com algum padrão pré-estabelecido. Tal padrão deverá ser adequado ao conjunto de respostas de cada questão norteadora, em particular.

A seguir, cada uma das questões será abordada, levando em conta a situação-problema apresentada anteriormente. 


\subsection{0 que deve ser feito?}

Começando com a pergunta "O que deve ser feito?", aplicada ao problema exposto, em primeiro lugar, é preciso explorar o seu verdadeiro significado. Essa tarefa deve contemplar os dois aspectos a seguir:

a) Leitura detalhada do enunciado, de modo a extrair os elementos necessários à compreensão dos fatos.

b) Listagem dos elementos extraídos.

Os elementos que serão extraídos podem se manifestar na forma de ações ou de dados. No caso em estudo, a leitura detalhada da situação-problema para a resposta da pergunta "O que deve ser feito?" pode ser conduzida, por exemplo, de acordo com o seguinte procedimento:

Cenário

- Primeiro parágrafo:

"Na obra de um edifício residencial com estrutura de concreto moldada no local e alvenaria de blocos de concreto, está sendo planejado um serviço de revestimento em uma parede”.

- Conclusão:

A finalidade desse parágrafo é apenas criar uma situação-problema.

- Segundo parágrafo:

"As dimensões da parede são $3 \mathrm{~m}$ de altura por $10 \mathrm{~m}$ de comprimento".

- Conclusão:

Existem dados concretos sobre o objeto em estudo, que são a altura e a largura da parede. Logo, existem duas ações de atribuição de dados, a saber:

i. Atribuir valor de $3 \mathrm{~m}$ para a altura da parede;

ii. Atribuir valor de $10 \mathrm{~m}$ para o comprimento da parede;

Questão:

Qual deverá ser a espessura da camada de gesso sabendo que não se poderá usar mais de cinquenta sacos de gesso, cada um pesando $25 \mathrm{~kg}$ ?

- Conclusão:

No intuito de responder a pergunta, deve-se considerar duas restrições do problema, que são resumidas nas ações de atribuições de dados:

iii. Atribuir 50 à quantidade de sacos de gesso;

iv. Atribuir $25 \mathrm{~kg}$ à massa de cada saco;

Formalmente, o que se necessita calcular é o valor com o qual ficará a espessura da camada de gesso, ação sintetizada como sendo:

Calcular a espessura do revestimento.

Reunindo os resultados das conclusões extraídas da situação-problema e da questão proposta, obtém-se uma síntese, que constitui a resposta da primeira pergunta fundamental, conforme o seguinte Quadro 1:

Quadro 1 - O que deve ser feito?
i. Atribuir valor de $3,0 \mathrm{~m}$ para a altura da parede;
ii. Atribuir valor de $10,0 \mathrm{~m}$ para o comprimento da parede;
iii. Atribuir 50 à quantidade de sacos de gesso;
iv. Atribuir $25 \mathrm{~kg}$ à massa de cada saco;
v. Calcular a espessura do revestimento. 
Cada uma das linhas representa uma síntese do que deve ser feito para se alcançar a resolução do problema. Uma prática recomendável nessa representação é a de que o começo da frase contenha sempre um verbo na forma infinitiva. Essa prática não é obrigatória, mas pode ser útil como uma forma de padronizar a expressão do raciocínio.

Assim, o que se está formulando, neste momento, é uma estrutura formal para a resolução da situação-problema por meio de uma visão geral do que precisa ser feito. Nesta fase, cada uma das linhas poderá apresentar algum grau de abstração, maior ou menor, de acordo com a percepção de quem formula e interpreta a situação-problema e a questão propostas.

\subsection{Como deve ser feito?}

A sequência de linhas no Quadro 1 é uma forma resumida de apresentar os procedimentos que devem ser dados para a resolução da situação-problema. Cada passo, ou instrução, se encontra expresso como uma frase, iniciada com verbo na forma infinitiva, que nem sempre revela aquilo que se deve realmente executar. Tal é o caso da instrução (v.) iniciada pelo verbo "calcular", cuja simples citação não indica exatamente que tipo de cálculo deve ser executado. Por outro lado, as instruções desde (i.) até (iv.) são iniciadas pelo verbo "atribuir", cujo sentido é mais restrito do que o do verbo "calcular". No entanto, mesmo nesses casos, é preciso estabelecer quem receberá a atribuição e o que vai ser atribuído. Então, resta definir elementos variáveis que fazem parte do problema, cada um recebendo o seu devido valor.

Começando pela instrução (i.), pode-se denominar a variável que representa a altura da parede por meio da forma mnemônica "Alt". A atribuição do valor $3 \mathrm{~m}$ à essa variável permitirá apresentar a instrução (i.) com a forma "Alt = $3 \mathrm{~m}$ ".

Para as outras instruções, até a (iv.), poderiam ser definidas as variáveis "Compr $=10 \mathrm{~m}$ ", "Sacos $=50$ " e "Massa Unitária $=25 \mathrm{~kg}$ ". Reunindo as novas formas das instruções de atribuição, o Quadro 1 toma a forma do Quadro 2, mostrado a seguir:

Quadro 2 - Como deve ser feito?

\begin{tabular}{|ll|}
\hline i. & Alt $=3,0 \mathrm{~m} ;$ \\
ii. & Compr $=10,0 \mathrm{~m} ;$ \\
iii. & Sacos $=50 ;$ \\
iv. & Massa Unitária $=25 \mathrm{~kg} ;$ \\
v. & Calcular a espessura do revestimento $(x)$. \\
\hline
\end{tabular}

Lembrar que os valores atribuídos às variáveis precisam ser seguidos das unidades de medida correspondentes. Este é um aspecto indispensável para que se consiga obter uma correta resolução do problema.

Falta ainda tratar da instrução (v.), cujo verbo "calcular" não torna claro como isso deve ser feito. Neste ponto, é preciso recorrer ao conhecimento anterior de Matemática, bem como aos dados técnicos do problema.

De acordo com o enunciado do problema, sabe-se que a matéria-prima para o revestimento da parede é o gesso, adquirido na forma de pó. Naturalmente, não se pode apenas espalhar o pó em sua forma original pela superfície da parede e esperar que o mesmo permaneça ali fixado. É necessário misturar o pó com água de modo a preparar um material aglutinante para o revestimento que, em seguida, será aplicado na parede. O resultado desse serviço é uma "camada de pasta de gesso" com três dimensões, comprimento, altura e espessura, que definem um volume de gesso aplicado. Em resumo, o processo consiste em receber uma massa de gesso e devolver um volume de revestimento, o que requer a utilização de um modelo matemático que relacione essas duas grandezas.

Do conhecimento da Física elementar, sabe-se que a Massa de um corpo é igual ao produto do Volume do mesmo pela Densidade do material que o compõe, o que pode ser escrito em linguagem matemática do seguinte modo:

Massa $=$ Volume ${ }^{*}$ Densidade.

Conforme o Quadro 2, o máximo de massa de gesso permitida (M) é aquela contida em cinco sacos. Então, concluise que a massa do material a ser usado é igual a:

$$
M=50 * 25 \mathrm{~kg}=1250 \mathrm{~kg}
$$


Esse valor é o que deve aparecer no lado esquerdo da expressão (1). Quanto aos dois elementos do membro direito, isto é, "Volume" e "Densidade", é preciso observar aspectos da situação e dos dados técnicos que acompanham a situação-problema.

Quando se apresenta na forma de pó, o gesso possui uma propriedade denominada Densidade Aparente $\left(\rho_{\mathrm{a}}\right)$, que é uma medida da densidade do material considerando o volume de grãos mais os espaços vazios entre eles. Desse modo, pode-se calcular o Volume do Gesso em Pó $\left(\mathbf{v}_{\mathbf{g}}\right)$ por meio da expressão

$$
\mathbf{v}_{\mathbf{g}}=\mathbf{M} / \rho_{\mathrm{a}}
$$

Outra propriedade importante é a chamada Densidade Absoluta $\left(\rho_{\mathrm{b}}\right)$, que se trata de uma medida de densidade obtida quando se suprimem os espaços vazios existente entre os grãos do gesso. Essa supressão pode ocorrer por meio de algum processo físico, por exemplo, a compressão do material.

Adicionando-se água ao material, observa-se uma alteração no volume e a consequente interferência no rendimento resultante, daí surge a necessidade de utilizar um fator para converter o volume do pó no volume da pasta de gesso, que recebe a denominação de Coeficiente de Rendimento (r) (VERSOZA, 1987) e se trata de um dado técnico do material. A expressão para o cálculo desse coeficiente foi estabelecida a partir de resultados experimentais como sendo a seguinte:

$$
\mathbf{r}=\left(\rho_{\mathrm{a}} / \rho_{\mathrm{b}}\right)+\text { Água_de_Amassamento }
$$

Além dos dois elementos que representam valores de densidades, o item Água de Amassamento é um valor constante, também obtido de modo experimental.

Segue que a relação entre os dois volumes, o Volume do Gesso em Pó $\left(\mathbf{v}_{\mathbf{g}}\right)$ e o Volume da Pasta de Gesso $\left(\mathbf{v}_{\mathbf{p}}\right)$ é a seguinte:

$$
\mathbf{v}_{\mathbf{p}}=\mathbf{r} * \mathbf{v}_{\mathbf{g}}
$$

Assim, para se determinar a Espessura (x) da camada de revestimento, é preciso dividir o Volume da Pasta de Gesso pelo valor da Área da Parede (Alt * Compr), conforme a expressão a seguir:

$$
\mathbf{x}=\mathbf{v}_{\mathbf{p}} /(\text { Alt } * \text { Compr })
$$

O Quadro 3, a seguir, contém o detalhamento das etapas necessárias para responder à pergunta fundamental "O que deve ser feito?". A fim de tornar a leitura mais resumida, foram utilizadas as expressões sucintas, começando pela (2) e indo até a expressão (6):

Quadro 3 - Como deve ser feito?

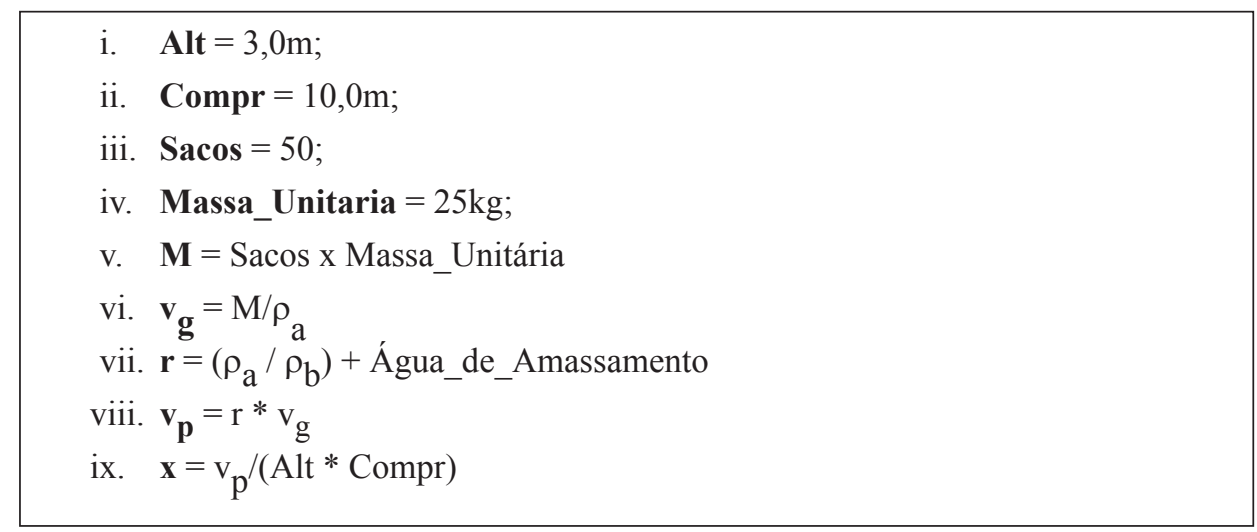

Dessa forma, o Quadro 3 contém a resposta para a segunda pergunta fundamental, que corresponde ao roteiro a ser seguido para encontrar o resultado do problema. A próxima etapa do método é aquela que se refere à implementação da resolução proposta. 


\subsection{De que maneira implementar?}

A busca pelo resultado do problema proposto se completa nesta etapa, quando são obtidos os valores numéricos a partir da sequência de passos construída na etapa anterior. Devem ser atribuídos nas expressões do Quadro 3 os valores das constantes e, em consequência, calculados os valores das variáveis, formando assim o que se encontra no Quadro 4, apresentado a seguir.

A atribuição dos valores das constantes dá origem ao Quadro 4:

Quadro 4 - De que maneira implementar?

\begin{tabular}{|ll|}
\hline i. & Alt $=3,0 \boldsymbol{m}=30,0 \mathrm{dm}$ \\
ii. & Compr $=10,0 \mathrm{~m}=100,0 \mathrm{dm}$ \\
iii. & Sacos $=50$ \\
iv. & Massa_Unitária $=25 \mathrm{~kg}$ \\
v. & M= $5 \times 25 \mathrm{~kg}=1250 \mathrm{~kg}$ \\
vi. & $\boldsymbol{v g}=1250 \mathrm{~kg} / 0,84 \mathrm{~kg} / \mathrm{dm}^{3}=1488,09 \mathrm{dm}^{3}$ \\
vii. & $\boldsymbol{r}=\left(0,84 \mathrm{~kg} / \mathrm{dm}^{3} / 2,7 \mathrm{~kg} / \mathrm{dm}^{3}\right)+0,450$ \\
\hline
\end{tabular}

Efetuando as operações aritméticas indicadas desde a linha (v.) até a linha (ix.), o problema está resolvido, obtendose o valor da espessura do revestimento aplicado na parede, conforme verificado na linha (ix.).

\section{Conclusão}

Em síntese, o professor, como condutor do processo educacional, precisa adotar métodos de ensino que estimulem o estudante, motivando-o a utilizar sua criatividade na resolução de problemas que envolvem sua realidade e campo de interesse.

Para tanto, neste artigo, introdutoriamente, apresentou-se um método para a resolução de problemas demonstrando sua aplicação com base em um caso real na Engenharia Civil. O método proposto segue uma estratégia pela qual se constrói um caminho lógico, desde a apresentação do problema até a solução final buscada.

Com base no exposto, espera-se que o método proposto, denominado pelos autores como MODULA(Uma abordagem estruturada para a resolução de problemas, aplicada ao ensino de Matemática na Engenharia) seja uma estratégia de ensino e aprendizagem viável e de fácil utilização por estudantes e professores.

Desse modo, busca-se difundir o método proposto, formalizando-o para objeto de análise em futuras pesquisas, visando sua adoção não apenas em cursos de Engenharia, mas em qualquer curso e disciplina que requeiram a organização lógica e sistemática de dados e procedimentos para a resolução de um problema de natureza quantitativa e/ou qualitativa.

Em artigo futuro, os autores analisarão a consistência e validade do método proposto e suas teorias subjacentes através dos polos da prática metodológica (BRUYNE et ali, 1982).

Finalmente, para clarificar, "método" e "processo" são dois conceitos que não se confundem, pois método é um dispositivo ordenado, um procedimento sistemático, em plano geral; enquanto que processo (ou técnica) é a aplicação e a forma específica de executar o método. Comparando-se os dois termos é possível afirmar que a relação existente entre método e processo é similar à existente entre "estratégia" e "tática". O processo está subordinado ao método e, ao mesmo tempo, é auxiliar fundamental do método.

Portanto, no contexto deste artigo, busca-se responder a questão norteadora introdutória (O que deve ser feito para organizar o trabalho pedagógico de forma a propiciar o desenvolvimento das competências e habilidades em Matemática?) com a proposição do construto científico denominado de MODULA, como um conjunto de processos que estruturam o método proposto, ao buscar responder: $O$ que deve ser feito? Como deve ser feito? e, finalmente, De que maneira implementar? 


\section{Referências}

ALMEIDA, C. S. de; GONTIJO, C. H. Dificuldades de aprendizagem em matemática e a percepção dos professores em relação a fatores associados ao insucesso nesta área. 2006. 13f. Trabalho de Conclusão de Curso (Graduação) - Universidade Católica de Brasília, Brasília, 2006.

AUSUBEL, D. P. The psychology of meaningful verbal learning. New York: Grune and Stratton, 1963.

BRUYNE, P.; HERMAN, J.; SCHOUTHEETE, M. Dinâmica da pesquisa em ciências sociais: os polos da prática metodológica. Rio de Janeiro: Francisco Alves, 1982.

DROMEY, R. G. How to solve it by computer. New Jersey: Prentice-Hall, 1982.

HADJI, C. A avaliação, regras do jogo: das intenções aos instrumentos. 4. ed. Porto: Porto Editora, 1994.

HUME, D. Dúvidas céticas relativas às operações do entendimento. In: Investigações sobre o entendimento humano e sobre os princípios da moral. São Paulo: UNESP, 2004. p. 53-70.

MACHADO, D. de Q. et al.. O modelo metodológico quadripolar de Bruyne, Herman e Schoutheete e as pesquisas qualitativas dos fenômenos sociais. Atas - Investigação Qualitativa em Educação, v. 1, 2016. p. 276-285.

MOREIRA, M. A. Teorias de aprendizagem. São Paulo: EPU, 1999. 195 p.

POLYA, G. How to solve it. New Jersey: Princeton University Press, 1945.

VERSOZA, E. J. Materiais de construção. São Paulo: Sagra, 1987.

VIANA, M. M. Programação de computadores para ciências e engenharia. Fortaleza: UFC, 2001.

VYGOTSKY, L. S. A formação social da mente. 2. ed. São Paulo: Martins Fontes, 1988.

Pensamento e linguagem. São Paulo: Martins Fontes, 1987.

YIN, Robert K. Estudo de caso: planejamento e métodos. Tradução de Daniel Grassi. 2. ed. Porto Alegre: Bookman, 2001.

Sobre os autores

\section{Mateus Mosca Viana}

Doutor em Engenharia de Teleinformática e Mestre em Matemática pela Universidade do Ceará - UFC e Professor de Engenharia de Computação pela Universidade Federal do Ceará - UFC, Professor da Universidade de Fortaleza UNIFOR.

\section{Ana Teresa de Araújo Farias}

Mestrado Matemática pela Universidade Federal do Ceará - UFC. Professor da Universidade de Fortaleza - UNIFOR.

Francisco Antonio de Araújo e Souza

Doutor em Educação pela Universidade Federal do Ceará - UFC, Mestrado em Engenharia de produção pela Universidade Federal do Amazonas - UFAM - Graduação em Ciências Econômica - Universidade do Amazonas - UNAM. 